\title{
Compensation For Bunch Emittance In A Magnetization And Space Charge Dominated Beam
}

\author{
Xiangyun Chang, Ilan Ben-Zvi, Jörg Kewisch \\ Collider-Accelerator Department, Brookhaven National Laboratory \\ Upton NY 11973 USA
}

\begin{abstract}
In order to obtain sufficient cooling rates for the Relativistic Heavy Ion Collider (RHIC) electron cooling, a bunched beam with high bunch charge, high repetition frequency and high energy is required and it is necessary to use a "magnetized" beam, i.e., an electron beam with non-negligible angular momentum. Applying a longitudinal solenoid field on the cathode can generate such a beam, which rotates around its longitudinal axis in a field-free region. This paper suggests how a magnetized beam can be accelerated and transported from a RF photocathode electron gun to the cooling section without significantly increasing its emittance. The evolution of longitudinal slices of the beam under a combination of space charge and magnetization is investigated, using paraxial envelope equations and numerical simulations. We find that we must modify the traditional method of compensating for emittance as used for normal non-magnetized beam with space charge to account for magnetization. The results of computer simulations of successful compensation are presented. Alternately, we show an electron bunch density distribution for which all slices propagate uniformly and which does not require emittance compensation.
\end{abstract}

\section{INTRODUCTION}

The average luminosity of the RHIC over 10 hours is many times smaller than its initial peak luminosity because of the increase in transverse- and longitudinalemittances due to Intra Beam Scattering (IBS), beam-beam effects, and external noise. Electron cooling may be the most effective way to suppress or reverse the growth of the beam's emittance, and may even increase peak luminosity.

As the electron beam energy is high for RHIC e-cooling a strong cooling solenoid is necessary to have enough cooling. When the beam enters or leaves the cooling solenoid, its angular momentum changes. To ensure zero angular momentum inside the cooling solenoid, an equivalent longitudinal magnetic field must be applied at the cathode according to Busch's theorem. The beam emerging from the cathode is deemed "magnetized". This angular momentum significantly changes the dynamics of the electron beam and can lead to large growth of emittance. The purpose of this work was to find a solution to beam optics that minimizes this growth, which is characterized by strong space-charge forces and angular momentum. 


\section{EMITTANCE COMPENSATION FOR A REGULAR NON- MAGNETIZED BEAM}

The emittance-compensation mechanism for a non-magnetized beam is discussed in many papers [2] [4] [5]. We start from the general paraxial envelope equation [1]:

$$
r_{m}^{\prime \prime}+\frac{\gamma^{\prime} r_{m}^{\prime}}{\beta^{2} \gamma}+\frac{\gamma^{\prime \prime} r_{m}}{2 \beta^{2} \gamma}+\left(\frac{q B}{2 m c \beta \gamma}\right)^{2} r_{m}-\left(\frac{p_{\theta}}{m c \beta \gamma}\right)^{2} \frac{1}{r_{m}^{3}}-\left(\frac{\varepsilon_{n}}{\beta \gamma}\right)^{2} \frac{1}{r_{m}^{3}}-\frac{K}{r_{m}}=0
$$

Where we assume that all the particles have the same $\beta$ and $\gamma$, and that the bunch length to diameter ratio in its rest frame is much larger than 1 . Here $r_{m}$ is the beam radius, $\mathrm{B}$ is the solenoid field, and $\mathrm{p} \theta$ is the canonical momentum on the cathode: $p_{\theta}=z m r_{s}^{2} \dot{\theta}_{s}+q A_{\theta} r_{s} \approx q A_{\theta} r_{s}=q B_{s} r_{s}^{2} / 2$ with $\mathrm{B}_{\mathrm{s}}$ and $\mathrm{r}_{\mathrm{s}}$ being the longitudinal magnetic field and the beam's radius on the cathode, respectively. $\varepsilon_{\mathrm{n}}$ is the normalized thermal emittance. $K=\frac{I(\zeta)}{I_{0}} \frac{2}{\beta^{3} \gamma^{3}}$ is the generalized perveance and $\zeta$ is the longitudinal position to the center of the beam bunch. $I_{0}=17000 \mathrm{~A}$ is the Alfven current. The equation is applicable if the motion of the particles is laminar, i.e., the transverse space-charge force is linear and $\mathrm{r}$ is proportional to $\mathrm{r}_{\mathrm{m}}$. We define $\mathcal{M}=\frac{p_{\theta}}{m c}=\frac{q B_{s} r_{s}^{2}}{2 m c}$ as the magnetization of an electron. A beam with non-zero $\mathrm{B}_{\mathrm{s}}$ is called a magnetized beam. $\mathcal{M}$ is equivalent to the normalized thermal emittance term $\varepsilon_{\mathrm{n}}$ in (1).

In this model, the emittance is constant for a thin slice of the bunch (thin being significantly shorter in length compared to its bunch length), but the emittance of the whole bunch changes due to the slices' relative motion. Emittance compensation reduces this effect.

There is a self-consistent distribution in a beam bunch wherein $\mathrm{T}_{\mathrm{SC}}(\zeta, t)$ depends only on time, $t$ : an ellipsoid of revolution in $\zeta$-r, with a round transverse cross-section. The volume charge density is uniform inside the bunch. Then, ignoring the thermalemittance term, for non-magnetized beam, (1) can be expressed as:

$$
r_{m}^{\prime \prime}(\zeta, t)+D(t) r_{m}(\zeta, t)=0
$$

where $D(t)$ is the sum of all the factors of focusing or defocusing terms in (1) and is the same for all slices. Accordingly, emittance compensation is not required for an ellipsoid distribution.

For a magnetized beam, we note that the $5^{\text {th }}$ term in (1) is proportional to $r_{m}(\zeta, 0)$ on cathode. Thus, for an ellipsoid distribution, Equation (2) will still be valid at some short distance from the cathode. In this case, compensation for emittance is not necessary either.

For a space charge dominated beam, $K r_{m}^{2}$ is much larger than $\frac{\varepsilon_{n}^{2}}{\beta^{2} \gamma^{2}}=\varepsilon^{2}$. In a drift space, equation (1) for a slice simply becomes:

$$
r_{m}^{\prime \prime}(\zeta)-K(\zeta) / r_{m}(\zeta)=0
$$

The solution [6] of (3) is: 


$$
Z=2 e^{\mp R_{0}^{\prime 2}} \cdot \int_{R_{0}^{\prime} \cdot}^{\mp \ln R+R_{0}^{\prime 2}} e^{\mp R_{0}^{\prime 2}} d R^{\prime} .
$$

Where $\mathrm{Z}$ and $\mathrm{R}$ are the generalized parameters: $Z=(2|K|)^{1 / 2} \frac{Z}{r_{0}}, R=\frac{r_{m}}{r_{0}}$.

The curves are shown in fig. 1 for a particle's different initial radial slopes. The larger the initial convergence angle, the earlier the beam form waist. Approximately at symmetric position to the waist, the divergence angle is the same magnitude of its initial convergence angle. If we assume that the positions of all the slice waists are close, we will have a small divergence angle spread between slices near the beam waist. This property is the basis for emittance compensation.

If the beam's thermal emittance is not small compared to $K r_{m}^{2}$, the envelope equation becomes:

$$
r_{m}^{\prime \prime}(\zeta)-\frac{\varepsilon^{2}}{r_{m}^{3}(\zeta)}-K(\zeta) / r_{m}(\zeta)=0
$$

The solution [7] of (5) is:

$$
Z=\int_{0}^{r_{m}}\left[r_{0}^{\prime 2}+\varepsilon^{2}\left(\frac{1}{r_{0}^{2}}-\frac{1}{r_{m}^{2}}\right)+2 K \ln \frac{r_{m}}{r_{0}}\right]^{-1 / 2} d r_{m} .
$$

The $\mathrm{R} \sim \mathrm{Z}$ curves are qualitatively similar to those in Fig.1.

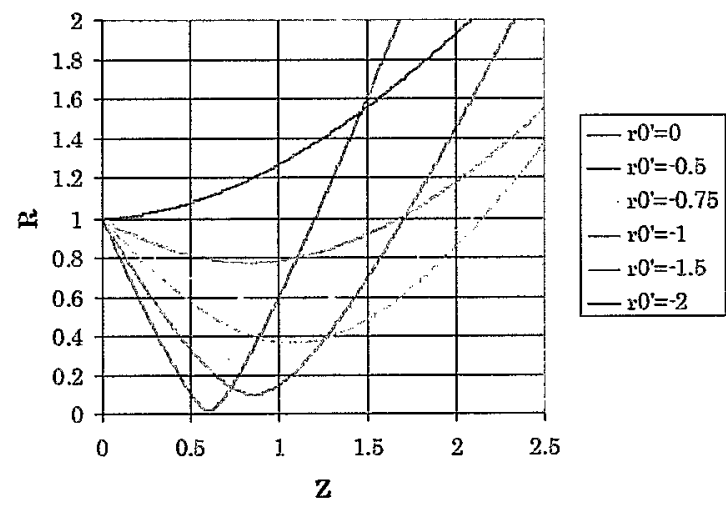

FIGURE 1. Envelope evolution for various initial angles

The emittance as a function of distance start from cathode is shown in fig. 2 which is the simulation of the BNL energy recovery linac (ERL) injection system with superconducting gun. There are 2 minima of emittance. The first one, near beam waist, corresponds to the state where the slope of low charge slice (line B) catches up that of high charge slice (line A). Generally this is the point where the emittance compensation is complete. After that, line B passes line A and the emittance increases. At some point, the slope of line A increases faster than the slope of line B because of its higher charge. The second minimum is at the point where line A re-catches up line B. The separation L of these two minima depends on the solenoid field, i.e., the beam size at waist. The larger the beam size at waist, the larger $\mathrm{L}$ will be. From the 
definition of $Z$ in fig.2, we also can see that we need a longer distance for higher charge beam to have the same emittance compensation effect as that of lower charge beam.

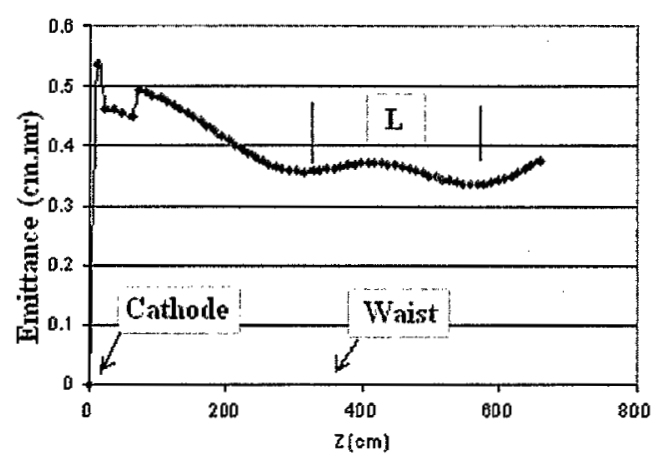

FIGURE 2. Simulation of ERL injection system with a gun followed by a solenoid and drift space, maximum field in gun is $30 \mathrm{MV} / \mathrm{m}$ and charge $=1 \mathrm{nC}$

\section{EMITTANCE COMPENSATION FOR A MAGNETIZATION AND SPACE-CHARGE DOMINATED BEAM}

When the beam's dynamics are dominated by both magnetization and space charge with ignorable thermal emittance $\varepsilon_{n}$, the paraxial envelope equation (1) for a slice in a drift space may be written as:

$$
r_{m}^{\prime \prime}-\left(\frac{\mathrm{M}}{\beta \gamma}\right)^{2} \frac{1}{r_{m}^{3}}-\frac{K(\zeta)}{r_{m}(\zeta)}=0 .
$$

The magnetization term introduces not only the beam's radial motion but also its angular motion. We define the emittance for the whole beam, $\varepsilon_{\mathrm{TO}}$, as the emittance measured in the frame rotating with an average rotation speed $\omega$. This is equivalent to the emittance in the laboratory frame by applying some adequate solenoid field, which is what we are really interested in for the electron-cooling application. We call this frame the $\langle\omega\rangle$ frame. If all the particles in this frame with same $\mathcal{M}$ have same radii they have no relative angular motion, then, by applying an appropriate solenoid field, the angular momentum can be set at zero in the laboratory frame. However, if beam particles with the same $\mathcal{M}$ have different radii, then, even after removing the average rotation, they will have some relative angular motion in laboratory frame. Hence, there are two new sources that contribute to the growth of emittance, $\varepsilon_{\mathrm{TOT}}$, for a magnetization-dominated beam: first, the relative change in the radii of slices caused by the dependence of $\mathrm{T}_{\mathrm{SC}}$ and $\beta \gamma$ on $\zeta$ and, second, the change in transverse distribution in each slice. The latter is small and will be neglected.

Our objective is to minimize the relative slice radii difference, while also minimizing the slice's spread of radial divergence before the emittances are 'frozen' by acceleration. 
The system is similar to that for non-magnetized beam emittance compensation but with the solenoid shifted towards the cathode to magnetize the beam (fig.3).

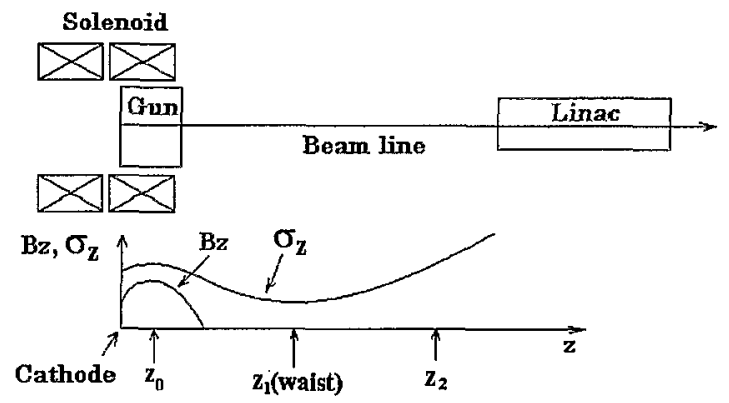

FIGURE 3. Emittance-compensation system for beam dominated by magnetization and space charge. $Z_{0}$ is the position of solenoid, $Z_{1}$ is the position of beam waist and $Z_{2}$ is the symmetric position of $Z_{0}$ to $Z_{1} \cdot Z_{2}-Z_{1}=Z_{1}-Z_{0}$

To simplify the analysis let's first assume that the particles have the same energy and at position $Z_{0}$ there is a short strong solenoid. The r-r' phase space propagation in the $\langle\omega\rangle$ frame is shown in fig. 4 .

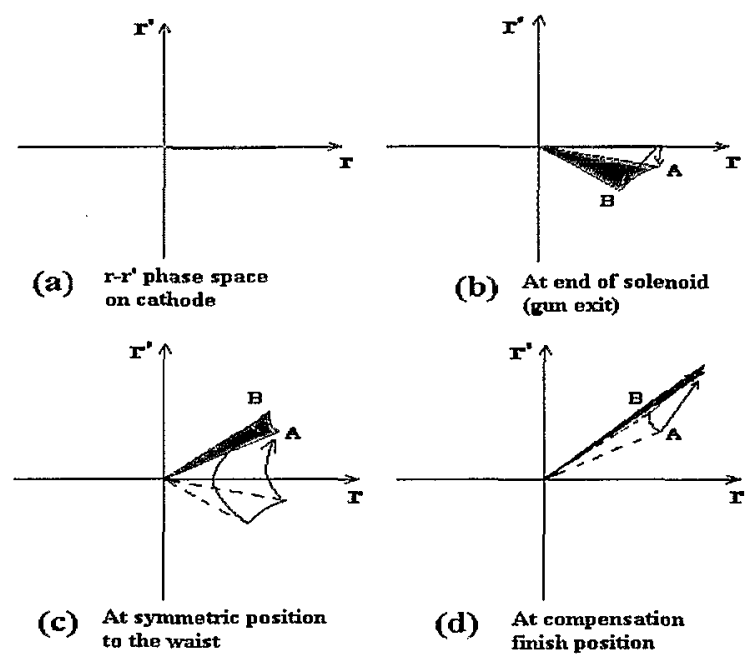

FIGURE 4. (a): r-r' phase-space on cathode. (b): at exit of solenoid. (c): after some drift space. (d): at compensation finish point

On cathode the beam is like a non-magnetized beam. The solenoid field is adjusted so that the beam is focused after the solenoid. Line $\mathrm{A}$ in fig. $4 \mathrm{~B}$ corresponds to the phase space of a high charge slice (middle of the bunch) at solenoid end while line B corresponds to a low charge slice (both ends of bunch). Because of the envelope propagation properties shown in fig. 1 the shape of the phase space is inverted about $r$ axis approximately at $Z_{2}$, i.e. line $B$ has a smaller radius, but larger slope (fig.4C). As the beam continues to drift the radius of line $B$ will catch up that of line $A$. Meanwhile, as line A has higher charge, its slope increases faster than that of line B if their radii are the same. At some point past $Z_{2}$ both the radius difference and the r-r' 
slope difference are minimized (fig.4D). The emittance as a function of distance is schematically shown in fig.5.

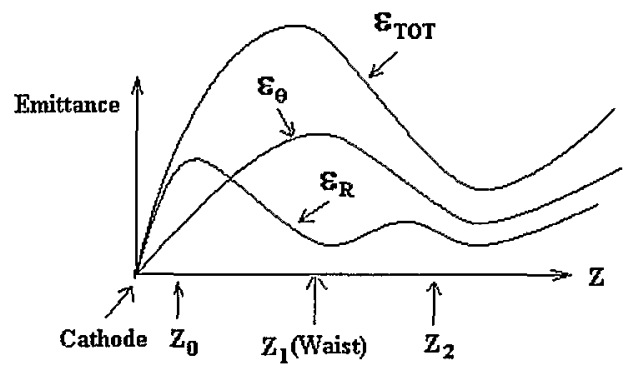

FIGURE 5. $\varepsilon_{\theta}$ is the emittance caused by slice relative angular motion, $\varepsilon_{\mathrm{R}}$ is the emittance caused by envelope divergence spread of slices. $\varepsilon_{T O T}=\sqrt{\varepsilon_{\theta}^{2}+\varepsilon_{R}^{2}}$ is the total emittance.

For non-magnetized beam we only have to consider $\varepsilon_{\mathrm{R}}$. When the slope of low charge slice catches up that of high charge near the waist at $Z_{1}$, the compensation is complete. For magnetized beam $\varepsilon_{\mathrm{TOT}}$ may be dominated by $\varepsilon_{\theta}$ and the slope of low charge slice must surpass that of high charge slice to compensate $\varepsilon_{\theta}$. Meanwhile the slope of high charge re-catches up that of low charge slice at the second minimum of $\varepsilon_{R}$. To have best compensation these two processes should complete simultaneously. The position of the minimum of $\varepsilon_{\theta}$ is later than $Z_{2}$. When the solenoid is too weak, i.e., $Z_{1}$ is too far to $Z_{0}$, before the radii difference compensation completes the slope of high charge slope already catches up that of low charge. On the contrary, if $Z_{1}$ is too close to $Z_{0}$, the slope of high charge slice will never catch up that of the low charge slice because that the radii increases quickly after $Z_{2}$ and the space charge term decreases quickly. Although there is no analytical solution of the solenoid strength to give best compensation, there must exist a unique solution for the best compensation. We can also conclude that the best compensation position is later than $Z_{2}$ in case $\varepsilon_{\theta}$ is dominated.

From the analysis above we see the differences between the compensation for nonmagnetized beam and the compensation for magnetized beam: For non-magnetized beam the compensation complete position is near beam waist $Z_{1}$ while for magnetized beam it is later than $Z_{2}$ and is near the second minimum of $\varepsilon_{R}$.

- For non-magnetized beam the compensation complete position is near beam waist $Z_{1}$ while for magnetized beam it is later than $Z_{2}$ and is near the second minimum of $\varepsilon_{R}$.

- For non-magnetized laminar beam there are multiple solutions for best compensation while for magnetized beam there exist only one solution.

The chromaticity effect can be considered as space charge effect to the $1^{\text {st }}$ order approximation. So, the above analysis can be applied but we need take the linac entrance focusing effect into account [8].

As an example Fig.6 shows the beam envelope as a function of distance from cathode to linac exit for optimized simulation of RHIC e-cooling electron injector. It's clear that the beam waist is far in front of the linac entrance. 


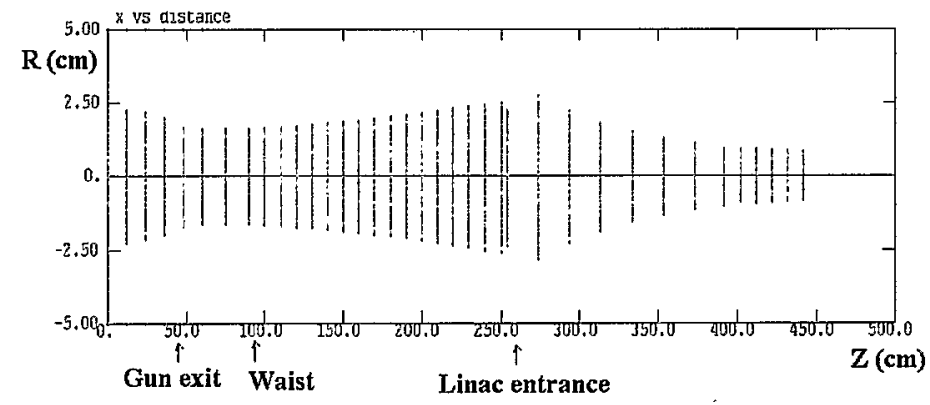

FIGURE 6. Beam envelope as a function of distance. Peak field on cathode $E_{c}=9 \mathrm{MV} / \mathrm{m}$ for a $2+1 / 2$ cell $700 \mathrm{MHz}$ photoinjector $\mathrm{RF}$ gun is assumed. Charge per bunch $=10 \mathrm{nc}$. On cathode $\mathrm{R}=1.5 \mathrm{~cm}, \mathrm{~B}=100$ Gausses. The magnetization $\mathcal{M}=660 \mathrm{~mm} . \mathrm{mr}$, therefore $\varepsilon_{\theta}$ will be dominating

\section{SUMMARY}

We analyzed the mechanics of emittance compensation for a magnetized beam with space charge. The layout of the accelerator is similar for both magnetized and nonmagnetized beams. The magnetization introduces a new degree of freedom, the $\theta$ direction motion. We find that for magnetization and space charge induced emittance increase the emittance complete position is far later than the beam waist and there is a unique solution for the solenoid strength. By approaching the laser's distribution to ellipsoid shape emittance can be greatly improved.

\section{REFERENCES}

1. Martin Reiser, Theory and Design of Charged Particle Beams, P210,. 1994 by John Wiley \& Sons, Inc. Printed in USA. ISBN 0-471-30616-9.

2. B. E. Carlsten, New Photoelectric Injector Design for The Los Alamos National Laboratory XUV FEL Accelerator, NIM in Physics Research, A285 (1989), P313-319.

3. O. D. Kellogg, Foundations of Potential Theory, P194,. 1967 by Springer Verlag. Berlin, New York.

4. Luca Serafini, Envelope Analysis of Intense Relativistic Quasi-laminar Beams in RF Photoinjectors: A Theory of Emittance Compensation, Physical Review E, Vol. 55, Number 6, P7565 7590. (1997).

5. S. G. Anderson and J. B. Rosenzweig, Non-equilibrium Transverse Motion and Emittance Growth in Ultrarelativistic Space-charge Dominated Beams, Physical Review Special Topics - Accelerators and Beams, Vol. 3, $094201(2000)$

6. Martin Reiser, Theory and Design of Charged Particle Beams, P199,. 1994 by John Wiley \& Sons, Inc. Printed in USA. ISBN 0-471-30616-9

7. Ibid., $\mathrm{P} 203$

8. Xiangyun Chang, Ilan Ben-Zvi, Jörg Kewisch, Design considerations for low field short photo-injected RF electron gun with high charge electronh bunch. Proceeding of the AAC04 meeting. 


\section{DISCLAIMER}

This report was prepared as an account of work sponsored by an agency of the United States Government. Neither the United States Government nor any agency thereof, nor any of their employees, nor any of their contractors, subcontractors or their employees, makes any warranty, express or implied, or assumes any legal liability or responsibility for the accuracy, completeness, or any third party's use or the results of such use of any information, apparatus, product, or process disclosed, or represents that its use would not infringe privately owned rights. Reference herein to any specific commercial product, process, or service by trade name, trademark, manufacturer, or otherwise, does not necessarily constitute or imply its endorsement, recommendation, or favoring by the United States Government or any agency thereof or its contractors or subcontractors. The views and opinions of authors expressed herein do not necessarily state or reflect those of the United States Government or any agency thereof.

\section{FOR UNCLASSIFIED, UNLIMITED STI PRODUCTS}

Available electronically at-

\section{OSTI:}

http://www.osti.gov/bridge

Available for a processing fee to U.S. Department of Energy and its contractors, in paper from-

U.S. Department of Energy

Office of Scientific and Technical Information

P.O. Box 62

Oak Ridge, TN 37831

(865) 576-8401

Facsimile: (865) 576-5728

E-mail: reports@adonis.osti.gov

\section{National Technical Information Service (NTIS):}

Available for sale to the public from-

U.S. Department of Commerce

National Technical Information Service

5285 Port Royal Road

Springfield, VA 22131

(800) 553-6847

Facsimile: (703) 605-6900

Online ordering: http://www.ntis.gov/ordering.htm 